\title{
Implementation of Machine Learning Approaches for Breast Cancer Prediction
}

\author{
Komal Hausalmal $^{1}$, J. P. Kshirsagar ${ }^{2}$ \\ komalhausalmal123@gmail.com¹,jpkshirsagar@gmail.com ${ }^{2}$
}

Article History: Received: 10 November 2020; Revised: 12 January 2021; Accepted: 27 January 2021; Published online: 05 April 2021 Abstract The grouping of bosom malignant growth has been the subject of enthusiasm for the fields of medicinal services
and bioinformatics, in light of the fact that it is the subsequent primary explanation of disease related passings in ladies.
Bosom malignancy can be investigated utilizing a biopsy where tissue is wiped out and concentrated under magnifying
instrument. The distinguishing proof of issue depends on the capability and experienced of the histopathologists, who will
consideration for unusual cells. Be that as it may, if the histopathologist isn't all around prepared or encountered, this
may prompt wrong finding. With the ongoing suggestion in picture handling and AI space, there is an enthusiasm for test to
build up a solid example acknowledgment based structure to improve the nature of finding. In this work, the picture highlight
extraction approach and AI approach is utilized for the grouping of bosom disease utilizing histology pictures into
threatening. The preprocessing on the picture is done using histopathological picture after that apply feature extraction and
classify the final result using SVM and Naive Bayes Classification techniques.

Keywords: Histopathological picture order, bosom disease analyze, feature extraction, SVM classification, Naive Bayes Classification.

\section{Introduction}

\section{A. Background}

Bosom malignant growth is the most widely recognized and risky meddling disease in ladies and the subsequent principle impact of malignancy passing in ladies, after lung malignant growth. The International Agency for Research on Cancer (IARC), which is a piece of the World Health Organization (WHO), the quantities of passings contemplated by malignant growth in the time of 2012 just come to around 8.2 million. The quantity of new cases is relied upon to development to in excess of 27 million by 2030.

Discovering bosom disease brisk and getting cutting edge malignant growth treatment are the key strategy to stay away from passings from bosom malignancy. In existing, it is a generally utilized approach to ID of bosom malignant growth by recognizing hematoxylin and eosin (HE) recolored histological slide arrangements that are checked under a powerful magnifying lens of the changed region of the bosom. In clinical practice, characterization of bosom malignancy biopsy result into various plans (for example harmful and noncancerous) is physically determined by experienced pathologists. Come out AI draws near and expanding picture volume created programmed framework for bosom malignant growth characterization conceivable and can assist pathologists with obtaining exact recognizable proof of issue increasingly effective.

Bosom malignant growth can be find or recognized utilizing clinical pictures testing utilizing histology and radiology pictures. The radiology pictures search can assist with finding the regions where the thing that matters is found. In any case, they can't be utilized to discover or recognized whether the region is dangerous. The biopsy, where a tissue is gives as information and prepared under a magnifying lens to check whether malignant growth is available, is the main sure approach to discover if a territory is dangerous. In the wake of finishing the biopsy, the distinguishing proof of issue will be founded on the capability of the histopathologists, who will break down the tissue under a magnifying instrument, searching for uncommon or carcinogenic cells. The histology pictures permit us to separate the cell cores types and their flowchart as per a particular example. Histopathologists especially inspect the consistency of cell shapes and tissue conveyances and chose the destructive districts and harm degree. On the off chance that the histopathologists are not all around prepared, this may prompt a mistaken recognizable proof of issue. Additionally, there is an absence of experts, which keep up the tissue test on hold for as long as two months. There is additionally the issue of reproducibility, as histopathology is an emotional science. This is correct particularly between non-specific pathologists, where we can get an alternate recognizable proof of issue on a similar example. Consequently, there is a persistent interest for PC helped distinguishing proof of issue.

\section{Review of Literature}

Breast cancer (BC) is a savage disease, executing a huge number of individuals consistently. Creating robotized dangerous $\mathrm{BC}$ recognition framework connected on patient's symbolism can assist managing this issue all the more effectively, making diagnosis more versatile and less inclined to mistakes. DeCAF (or profound) features contain an in course of action it relies upon reusing a some time ago prepared CNN similarly as feature 
vectors, which is then used as commitment for a classifier arranged only for the new request task. In the light of this, they show an appraisal of DeCaf features for $\mathrm{BC}$ acknowledgment, with a particular ultimate objective to all the almost certain perceive how they appear differently in relation to exchange systems [1].

This work proposes to order bosom malignant growth histopathology pictures autonomous of their amplifications utilizing convolutional neural systems (CNNs). They propose two unique designs; single assignment $\mathrm{CNN}$ is utilized to foresee harm and perform various tasks CNN is utilized to anticipate both threat and picture amplification level all the while. Assessments and correlations with past outcomes are completed on BreaKHis dataset [2].

The reason for this work is to create an insightful remote discovery and finding approach for breast disease in light of cytological pictures. At first, this work shows a totally motorized procedure for cell cores acknowledgment and division in chest cytological pictures. The territories of the cell centers in the image were related to indirect Hough change. The ejection of bogus positive (FP) revelations (noisy circles and platelets) was accomplish using Otsu's thresholding system and cushioned c-infers gathering technique. The division of as far as possible was capable with the usage of the marker-controlled watershed change. Next, an insightful bosom danger gathering structure was made [3].

The adequacy of the treatment of bosom disease relies upon its ideal recognition. An early development in the finding is the cytological assessment of bosom material obtained clearly from the tumor. This work gives in PC upheld bosom development conspicuous verification of issue considering the assessment of cytological pictures of fine needle biopsies to perceive this biopsy as either altruistic or destructive. Instead of give on the specific division of cell cores, the cores are finding by circles using the indirect Hough change framework. The outcome circles are then filtered to save simply astonishing estimations for also consider by an assist vector with machining which gatherings distinguished circles as right or wrong using surface features and the degree of centers pixels according to a centers shroud obtained using Otsu's thresholding framework [4].

This work direct some fundamental examinations utilizing the deep learning way to deal with arrange breast cancer histopathological pictures from BreaKHis, an openly dataset accessible at http://webinf.ufpr.brivri/bosom malignancy database. They propose a system taking into account the extraction of picture patches for setting up the CNN and the blend of these patches for clear gathering. This procedure intends to allow using the significant standards histopathological pictures from BreaKHis as commitment to existing CNN, keeping up a vital good ways from changes of the model that can incite a progressively eccentric and computationally over the top designing [5].

Current philosophies rely upon handcraft feature depiction, for instance, concealing, surface, and Local Binary Patterns (LBP) in masterminding two zones. Diverged from deliberately gathered incorporate based philosophies, which incorporate endeavor subordinate depiction, DCNN is an end to-end feature extractor that may be clearly picked up from the unrefined pixel power estimation of EP and ST tissues in a data driven form.These abnormal state highlights add to the development of a directed classifier for separating the two kinds of tissues [6].

The test turns out to be the means by which to cleverly join fix level arrangement results and model the way that not all patches will be discriminative. They propose to prepare a choice combination model to total fix level forecasts given by fix level CNNs, which to the best of our insight has not been appeared previously. They apply the technique to the grouping of glioma and non-little cell lung carcinoma cases into subtypes [7].

Computerized atomic identification is a basic advance for various PC helped pathology related picture examination calculations, for example, for mechanized evaluating of breast disease tissue examples. Nevertheless, automated center area is tangled by (1) the gigantic number of cores and the proportion of significant standards digitized pathology pictures, and (2) the capriciousness in gauge, shape, appearance, and surface of the individual cores. Starting late there has been eagerness for the use of "Significant Learning" methods for request and examination of colossal picture data [8].

This work present a dataset of 7,909 bosom tumor (BC) histopathology pictures acquired on 82 patients, that is as of now transparently open from http://web.inf.ufpr.br/vri bosom malignant growth database. The dataset joins both benevolent and harmful pictures. The endeavor identified with this dataset is the robotized characterization of these photos in two classes, which would be a significant PC helped discovering instrument for the clinician. So as to evaluate the trouble of this undertaking, we demonstrate some primer outcomes acquired with state-of-the-art image classification systems [9]. 
There are a couple of issues despite everything exist in regular individual Breast Cancer Diagnosis. To deal with the issues, an individual credit evaluation show taking into account assist vector with requesting strategy is proposed. Using SPSS Clementine data mining gadget, the individual credit data is packing examination by Support Vector Machine. It is examined in detail with the particular part limits and boundaries of Support vector machine. Bolster vector machine could be utilized to enhance crafted by medicinal specialists in the determination of breast growth [10].

\section{Proposed Methodology}

Ordering bosom malignancy histopathological pictures naturally is a significant assignment in PC helped pathology investigation. In any case, separating educational and non- excess highlights for histopathological picture order is testing. In our proposed work using Histopathological image, firstly we will apply image preprocessing technique to remove the noise of an image. After that we will apply the feature extraction process. The element based methodologies comprise of the highlights extraction stage and afterward arrangement stage. This methodology centers around extricating the element of picture and characterize them utilizing AI grouping technique. The removed highlights are prepared utilizing bolster vector machines and Naive Bayes Classification strategy. At long last, we looked at the exhibition utilizing the current grouping techniques.

\section{Advantages of Proposed System:}

1. Work could be advantageous to get quick and exact measurement, lessen onlooker fluctuation, and increment objectivity.

2. Cell nuclei detection using image thresholding and image edge detection.

3. We can measure accurate cell features.

4. This application can be utilized by doctors from their homes or some other spot.

5. This work will be appropriate for pictures with a serious extent of clamor and platelets and cell covering, as it can effectively identify the phone cores.

6.

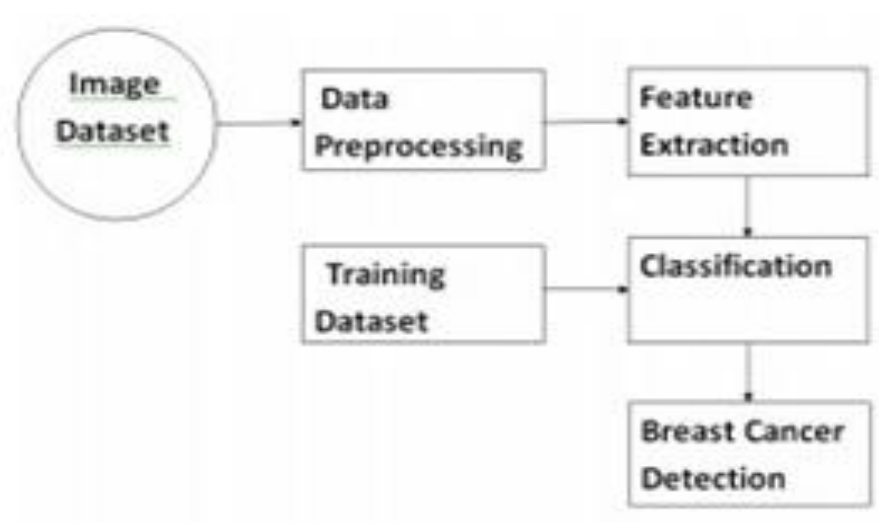

Figure1. Proposed System Architecture

\section{Explanation:}

\section{Input of system:}

In this proposed system, we take histopathological breast image as an input for processing.

Image Pre-processing:

In this step, check the size of input image and then the input image is converted into grayscale image. Also, we remove the noise of image using noise reduction technique that i.e. here we use the median filter for noise reduction.

Feature Extraction:

In this step, after image preprocessing, we extracts all feature of preprocessed image i.e. infected and healthy cell nuclei. Classification:

In this step, after image feature extraction, we classify the infected and healthy cell nuclei using support vector machine and also na"ive bays classification technique. 


\section{Result:}

This step displays the final breast cancer result.

\section{B. Algorithms}

\section{Support Vector Machine:}

Support Vector Machine (SVM) is utilized to arrange the natural product quality. SVM Support vector machines are essentially two class classifiers, direct or non-straight class limits.

The thought behind SVM is to shape a hyper plane in the middle of the informational indexes to communicate which class it has a place with.

The undertaking is to prepare the machine with known information and afterward SVM locate the ideal hyper plane which gives most extreme separation to the closest preparing information purposes of any class.

\section{Steps:}

Step 1: Read the test image features and trained features. Step 2: Check the all test features of image and also get all train features.

Step 3: Consider the kernel.

Step 4: Train the SVM using both features and show the output.

Step 5: Classify an observation using a Trained SVM Classifier.

\section{Näive Bays Classification:}

Naive Bayes calculation is the calculation that learns the likelihood of an article with specific highlights having a place with a specific gathering/class.In short,it is a probabilistic classifier.

The Naive Bayes calculation is classified "naive" since it makes the presumption that the event of a specific component is autonomous of the event of different highlights.

The Naive Bayesian classifier depends on Bayes' hypothesis with the autonomy surmise between indicators.

A Naive Bayesian model is anything but difficult to frame, with no basic iterative boundary calculation which makes it especially valuable for huge datasets.

Despite its effortlessness, the Naive Bayesian classifier frequently does especially well and is broadly utilized in light of the fact that it regularly beats increasingly experienced arrangement techniques.

\section{Mathematical Model}

\section{Mathematical Equations of Support Vector Machine:}

We have $\mathrm{k}$ sub-spaces so that there are $\mathrm{k}$ grouping consequences of sub-space to ordering bosom malignant growth cells, called CL SS1,CL SS2, ..., CL SSk. Thus the problem is how to integrate all of those results. Subsequently the issue is the way to incorporate those outcomes. The straightforward coordinating path is to compute the mean worth:

$$
\mathrm{CL}=\frac{1}{k} \sum_{i=1}^{k} C L_{-} S S_{i} \longrightarrow(1)
$$

Or weighted mean value:

$$
\mathrm{CL}=\frac{1}{k} \sum_{i=1}^{k} W_{i} C L_{-} S S_{i} \longrightarrow(2)
$$

Where $\mathrm{Wi}$ is the weight of classification result of subspace, i.e. breast cancer cells result, SSi and satisfies:

$$
\sum_{i=1}^{R} W_{i}=1
$$

The centroid is calculated as follows:

$$
\bar{X}=\frac{\sum_{i=0}^{k} X_{i}}{k}, \bar{Y}=\frac{\sum_{i=0}^{k} Y_{i}}{k}
$$


Where $(\mathrm{X}, \mathrm{Y}$ ) signifies the centroid of the hand, $\mathrm{Xi}$ and $\mathrm{Yi}$ are $\mathrm{x}$ and $\mathrm{y}$ coordinates of the ith pixel in the hand region and $\mathrm{k}$ denotes the number of histopathological image pixels that represent only the hand portion. In the next step, the distance between the centroid and the pixel value was calculated. For distance, the following Euclidean distance was used:

$$
\text { Distance }=\sqrt{(x 2-x 1)^{2}(y 2-y 1)^{2}}-(5)
$$

Where (x1, x2) and (y1, y2) represent the two co-ordinate values of histopathological image pixel.

\section{Mathematical equation in Naive-Bayes Classification:}

It gives us a strategy to figure the contingent likelihood of an occasion dependent on past information accessible on the occasions. Here we will use this technique for breast cancer classification. More formally, Byes Theorem is stated as the following equaction:

$$
\mathrm{P}\left(\frac{A}{B}\right)=\frac{P\left(\frac{B}{4}\right) P(A)}{P(B)}
$$

Let us comprehend the announcement first and afterward we will take a gander at the verification of the announcement. The segments of the above explanation are:

$\mathrm{P}(\mathrm{A} \mathrm{B})$ : Likelihood (conditional probability) of occurrence of event A given the event $\mathrm{B}$ is true

$\mathrm{P}(\mathrm{A})$ and $\mathrm{P}(\mathrm{B})$ : Probabilities of the incidence of event $\mathrm{A}$ and $\mathrm{B}$ respectively

$\mathrm{P}(\mathrm{B} \mathrm{A})$ : Likelihood of the incidence of event $\mathrm{B}$ given the event $\mathrm{A}$ is true

\section{A. Dataset}

This proposed framework use Breast malignant growth UCI AI dataset. Features are registered from a digitized picture of a fine needle suction (FNA) of a bosom mass. They depict attributes of the cell cores present in the picture. A couple of the pictures can be found at [Web Link] Separating plane portrayed above was acquired utilizing Multisurface MethodTree (MSM-T) [K. P. Bennett, "Choice Tree Construction Via Linear Programming."Proceedings of the 4th Midwest Artificial Intelligence and Cognitive Science Society, pp. 97-101, 1992], a organization method which uses linear programming to construct a decision tree. Applicable highlights were chosen utilizing a comprehensive inquiry in about 1-4 highlights and 1-3 isolating planes.

\section{Result and Discussion}

Tests will be finished by a PC with a design: Intel (R) Core (TM) i5-6700HQ CPU @ 2.60GHz, 16GB memory, Windows 8, MySQ1 Server 5.1 and Jdk 1.8. A few capacities utilized in the calculation are given by Opencv2.4.7. We will use the histopathological breast image data set which consist of total 500 images, collected from the 500 different patient of both healthy and infected. This data set collected from city hospital.

\section{Use of mean and Standard deviation:}

Standard deviation (SD) is a generally utilized estimation of changeability utilized in insights. It shows how much variety there is from the normal (mean). A low SD demonstrates that the information directs incline toward be near the mean, though a high SD shows that the information are spread out over an enormous scope of qualities.

The mean and standard deviation values of the input image are computed in each spectral channel as the feature. We let $\mathrm{n}$ be the number of pixels in the input image, and vij denotes the jth band value of the ith pixel in a image. The mean (meanj) and standard deviation (stdj) of the patch are calculated according to

$$
\begin{aligned}
& \text { Mean } J=\frac{\sum_{i=1}^{n} V_{i j}}{n} \\
& S t d_{j}=\sqrt{\frac{\sum_{i=1}^{n} V_{i j}-\operatorname{mean}_{j}^{2}}{n}}
\end{aligned}
$$

Table I is a summary of classification accuracies among different classifiers based on the feature for classifiers. Note that the Support Vector Machine and Na"ive Bayes-based classifier outperform other classifiers. 
The classification accuracy for SVM and NB is $77.5 \%$ and $77.2 \%$ on average, respectively.

Table 1. Table of mean and deviation table

\begin{tabular}{|l|l|l|l|l|}
\hline Classifier & $\begin{array}{l}\text { Mean } \\
\text { (Exis. } \\
\text { System) }\end{array}$ & $\begin{array}{l}\text { Standard } \\
\text { Dev. (Exis. } \\
\text { System) }\end{array}$ & $\begin{array}{l}\text { Mean } \\
\text { (Prop. } \\
\text { System) }\end{array}$ & $\begin{array}{l}\text { Standard } \\
\text { Dev. (Prop. } \\
\text { System) }\end{array}$ \\
\hline $\begin{array}{l}\text { Support Vector } \\
\text { Machine }\end{array}$ & 72.1 & 5.8 & 77.5 & 7.4 \\
\hline $\begin{array}{l}\text { Näive Bayes } \\
\text { Classification }\end{array}$ & 70.3 & 5.0 & 77.2 & 7.1 \\
\hline
\end{tabular}

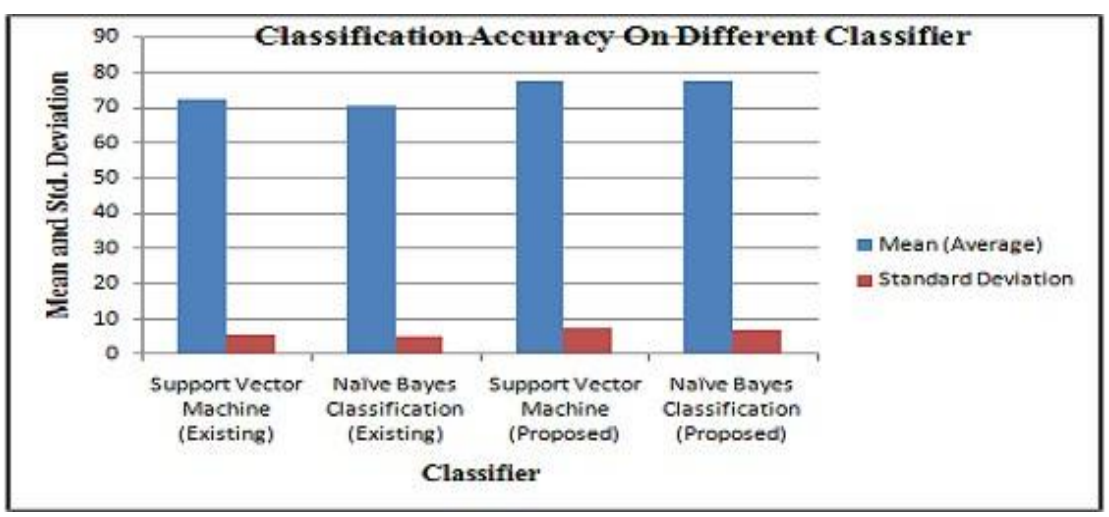

Figure 2. Mean and Deviation Graph

A. Performance Analysis

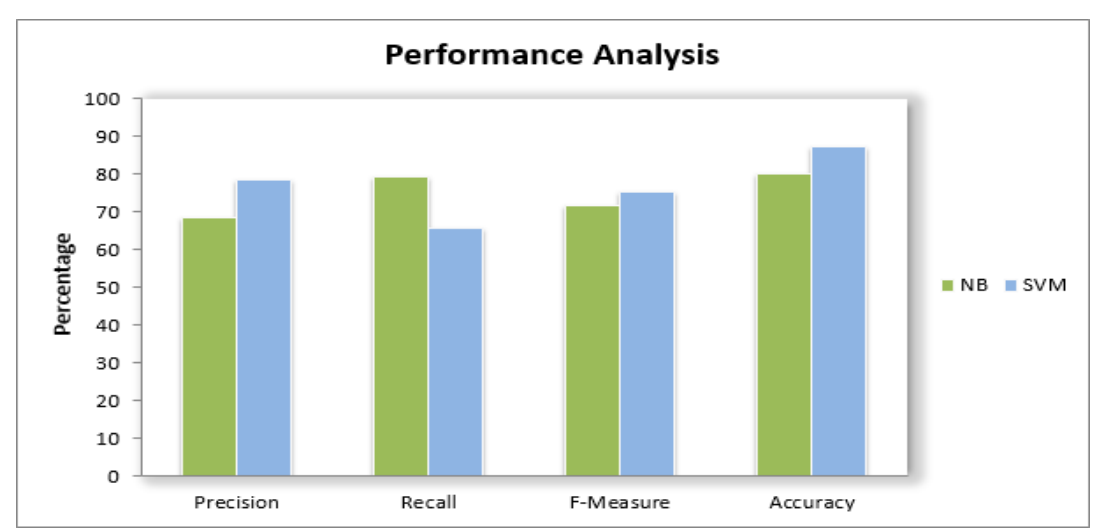

Figure 3. Accuracy Graph

Table 2. Accuracy table

\begin{tabular}{|l|r|r|}
\hline & Naiive Bayes & SVM \\
\hline Precision & 68.45 & 78.70 \\
\hline Recall & 79.44 & 65.64 \\
\hline F-Measure & 72.11 & 74.31 \\
\hline Accuracy & 80.29 & 87.26 \\
\hline
\end{tabular}

\section{Conclusion}

This proposed framework deal with histopathological pictures by utilizing Support Vector Machine (SVM) and Naive Bayes Classification with different arrangements for the order of bosom disease histology pictures into threat- ening. The designed SVM topology and Naive Bayes Classification worked well on histopathological images features in organization tasks. In any case, the presentation of the SVM arrangement and Naive Bayes 
Classification are bet- ter contrasted with the one of the current characterization techniques. SVM have become best in class, exhibiting a capacity to unravel testing order assignments. This proposed work effectively arranges utilizing bosom disease histology pictures into threatening.

\section{References}

1. F. A. Spanhol, L. S. Oliveira, P. R. Cavalin, C. Petitjean, and L. Heutte, "Deep features for breast cancer histopathological image classification," 2017 IEEE International Conference

2. N. Bayramoglu, J. Kannala, and J. Heikkila, "Deep learning for mag- nification independent breast cancer histopathology image classification," 2016 23rd International Conference on Pattern Recognition (ICPR), 2016.

3. Y. M. George, H. H. Zayed, M. I. Roushdy, and B. M. Elbagoury, "Remote computeraided breast cancer detection and diagnosis system based on cytological images," IEEE Systems Journal, vol. 8, no. 3, pp. 949-964, Sept 2014.

4. P. Filipczuk, T. Fevens, A. Krzyzak, and R. Monczak, "Computeraided breast cancer diagnosis based on the analysis of cytological images of fine needle biopsies," IEEE Transactions on Medical Imaging, vol. 32, no. 12, pp. 2169-2178, 2013.

5. F. A. Spanhol, L. S. Oliveira, C. Petitjean, and L. Heutte, "Breast cancer histopathological image classification using convolutional neural net- works," in International Joint Conference on Neural Networks, Vancouver, BC, Canada, July 2016, pp. 2560-2567.

6. J. Xu, X. Luo, G. Wang, H. Gilmore, and A. Madabhushi, “A deep convolutional neural network for segmenting and classifying epithelial and stromal regions in histopathological images," Neurocomputing, vol. 191, pp. 214-223, 2016.

7. L. Hou, D. Samaras, T. M. Kurc, Y. Gao, J. E. Davis, and J. H. Saltz, "Patch-based convolutional neural network for whole slide tissue image classification," in IEEE Conference on Computer Vision and Pattern Recognition, Las Vegas, Nevada, USA, June 2016, pp.2424-2433.

8. J. Xu, L. Xiang, Q. Liu, H. Gilmore, J. Wu, J. Tang, and A. Madabhushi, "Stacked sparse autoencoder (SSAE) for nuclei detection of breast cancer histopathology images," IEEE transactions on medical imaging, vol. 35, no. 1, pp. 119-130, 2016.

9. F. Spanhol, L. Oliveira, C. Petitjean, and L. Heutte, "A dataset for breast cancer histopathological image classification," IEEE Transactions on Biomedical Engineering, vol. 63, no. 7, pp. 1455-1462, 2016.

10. Shang Gao Hongmei Li, "Breast Cancer Diagnosis Based on Support Vector Machine". 2012 International Conference on Uncertainty Reason- ing and Knowledge Engineering

11. Su H, Liu F, Xie Y, Xing F, Meyyappan S, Yang L. "Region segmenta- tion in histopathological breast cancer images using deep convolutional neural network." In Biomedical Imaging (ISBI), 2015 IEEE 12th Inter- national Symposium on 2015 Apr 16 (pp. 55-58).

12. Wan, S., Huang, X., Lee, H.C., Fujimoto, J.G. and Zhou, C., 2015, April. "Spoke-LBP and ring-LBP: New texture features for tissue classification". In Biomedical Imaging (ISBI), 2015 IEEE 12th International Symposium on (pp. 195-199). IEEE.

13. Levi, Gil, and Tal Hassner. "Age and gender classification using con- volutional neural networks." In Proceedings of the IEEE Conference on Computer Vision and Pattern Recognition Workshops, pp. 34-42. 2015.

14. M. Veta et al., "Breast cancer histopathology imge analysis: A review," IEEE Transactions on Biomedical Engineering, vol. 61, no. 5, p. 1400, 14112014.

15. Desir et al., "Classification of endomicroscopic images of the lung based on random subwindows and extra-trees," IEEE Transaction on Biomedical Engineering, vol. 59, no. 9, pp. 2677-2683, 2012. 